\title{
Prognostic Value of Combined; Cox-2, Cyclin D1 and P21 Expression in Colorectal Cancer (CRC) Patients: An Immunohistochemical Study
}

\author{
Amir M. Salem1, Mariem A. Elfeky ${ }^{1 *}$, Nashwa Nawar², Ahmed Z. Alattar ${ }^{2}$, \\ Omar Atef Elekiabi ${ }^{3}$, Mostafa M. Elaidy ${ }^{3}$ \\ ${ }^{1}$ Department of Pathology, Faculty of Medicine, Zagazig University, Zagazig, Egypt \\ ${ }^{2}$ Department of Clinical Oncology and Nuclear Medicine, Faculty of Medicine, Zagazig University, Zagazig, Egypt \\ ${ }^{3}$ Department of General Surgery, Faculty of Medicine, Zagazig University, Zagazig, Egypt \\ Email: *Mariemelfeky@gmail.com
}

How to cite this paper: Salem, A.M., Elfeky, M.A., Nawar, N., Alattar, A.Z., Elekiabi, O.A. and Elaidy, M.M. (2018) Prognostic Value of Combined; Cox-2, Cyclin D1 and P21 Expression in Colorectal Cancer (CRC) Patients: An Immunohistochemical Study. Open Journal of Pathology, 8, 106-121.

https://doi.org/10.4236/ojpathology.2018.8

$\underline{3013}$

Received: June 22, 2018

Accepted: July 27, 2018

Published: July 30, 2018

Copyright (c) 2018 by authors and Scientific Research Publishing Inc. This work is licensed under the Creative Commons Attribution International License (CC BY 4.0).

http://creativecommons.org/licenses/by/4.0/

\section{(c) (i) Open Access}

\begin{abstract}
Background: Cyclooxygenase (COX) is a rate limiting enzyme in synthesis of prostanoids pathway and it has 2 isoforms (COX-1 and COX-2). It has many biological roles in inflammation and oncogenesis. Cox-2 was incriminated in performing disturbances in the cell cycle control in CRC, but its role of in CRC needs clarification of the mechanisms by which Cox-2 might affect the process of colorectal carcinogenesis. Cyclin D1 is an oncogene that regulates G1 phase progression to S phase of the cell cycle. Its stimulatory role on cell cycle is antagonized by Cyclin D1-dependent kinase (CDK) inhibitors like p21. P21 plays an essential role in cell cycle regulation; it may have a pro-apoptotic or an antiapoptotic role in cancer. P21 was found to have many roles in cancer; invasion metastases, cellular senescence and stem cells aging. The roles of combined expression of Cox-2, Cyclin D1 and P21 in CRC tissues and their role of prognosis and patients survival are not sufficiently clarified. Aim of the Study: To evaluate tissue expression of Cox-2, Cyclin D1 and P21 in CRC and to correlate such expression with pathological parameters, clinical and prognostic data of the patients. Methods: Cox-2, Cyclin D1 and P21 are evaluated in colon cancer tissues. Correlations between their level of expressions pathological parameters, clinical and prognostic data of patients were analyzed. Results: Cox-2, Cyclin D1 over-expression was associated with higher grade, higher incidence of occurrence of lymph node \& distant metastasis and advanced stage $(\mathrm{P}=0.000)$. Cox-2 was related to higher tumor recurrence rate $(\mathrm{P}=0.04)$ and decreased overall patients survival rate $(\mathrm{p}=$ 0.002). ( $\mathrm{r}$ correlation coefficient $=+0.987$ ). Conclusion: Cox -2 and Cyclin D1 are markers of poor prognosis colon cancer patients.
\end{abstract}




\section{Keywords}

Cox-2, Cyclin D1, Colon Cancer, Immunohistochemistry, Prognosis

\section{Introduction}

Colorectal cancer (CRC) is the $4^{\text {th }}$ commonest cancer related fatality and one of the commonest reasons of morbidity worldwide [1]. It is considered the $3^{\text {rd }}$ frequently occurred cancer worldwide [2]. Early diagnosis of such type of cancer leads to higher chance of adequate management, curative treatment and decrease its high metastatic rate [3]. Additionally its early detection by nationally designed programs of screening in populations that are lacking clinical signs and symptoms allowed reduction in its rate of occurrence and high fatality in the developed countries [4]. Moreover it will be helpful to detect more novel predictive, prognostic and therapeutic targets for that malignancy to design more recent therapeutic strategies so as to improve patients' prognosis and survival rate [5]. Cyclooxygenase (COX) is a rate limiting enzyme in synthesis of prostanoids pathway and it has 2 isoforms (COX-1 and COX-2). It has many biological roles in inflammation and oncogenesis [6].

Increased levels of COX-2 were found in response to various cytokines, growth factor, and cancer promoting factors [7]. Additionally Cox-2 had many roles in apoptosis, angiogenesis, proliferation, invasion and metastases of plethora of tumor types [8]. It was found that levels of tissue protein expression of Cox-2 in CRC are increased in comparison to normal mucosa which point to its role in progression of such malignancy [9]. Moreover COX-2 expression was upregulated in carcinomas of many organs e.g. ovary, lung and cervix and was associated with poor patients' prognosis [10]. Additionally increased tissue expression of COX-2 in gastric cancer is associated with higher grade and advanced stage [11]. Cox-2 was incriminated in performing disturbances in the cell cycle control in CRC, but its role of in CRC needs clarification of the mechanisms by which Cox- 2 could affect the process of colorectal carcinogenesis.

Cyclin D1 is an oncogene that regulates G1 phase progression to S phase of the cell cycle [5]. Its stimulatory role on cell cycle is antagonized by cyclin D1-dependent kinase (CDK) inhibitors like p27 and p21 [12]. Hence Cyclin D1 is a controlling factor of G1 progression in the cell cycle, so it supposed to play an essential role in malignant progression and carcinogenesis [13]. Cyclin D1 tissue protein expression has been detected in a plethora of malignancies including CRC [14]. The role of its expression in CRC has been extensively explored but no final established results were detected [15]. Progression of cell cycle requires sequences of activation and inhibition of cyclin-dependent kinases (CDKs) like P21 [16]. P21 plays an essential role in cell cycle regulation; it may have a pro-apoptotic or an antiapoptotic role in cancer. P21 was found to have many roles in cancer; invasion metastases, cellular senescence and stem cells ag- 
ing [15]. The roles of combined expression of Cox-2, Cyclin D1 and P21 in CRC tissues and their role of prognosis and patients survival are not sufficiently clarified.

Aim of the study; to evaluate tissue expression of Cox-2, Cyclin D1 and P21 in $\mathrm{CRC}$ and to correlate such expression with pathological parameters, clinical and prognostic data of the patients.

\section{Patients \& Methods}

Our study is a prospective cohort study that includes sections from formalin-fixed, paraffin embedded samples from 60 CRC patients. All CRC patients are operated in General Surgery Department, Faculty of Medicine, Zagazig University in the period from May 2015 to May 2018. Specimen sent, processed, diagnosed, graded and staged by 2 pathologists in Pathology Department, Faculty of Medicine, Zagazig University. Grading based on the degree of gland formation and staging based on lymph node and distant metastases. Patients' follow-up data all through the study is obtained from Department of Clinical Oncology and Nuclear Medicine, Faculty of medicine, Zagazig University.

This work was approved by the local Ethics Committee

\section{1) Immunohistochemical staining}

Immunohistochemistry was done as previously described [17]. We have incubated slides with primary rabbit polyclonal anti-COX2/Cyclooxygenase 2 (ab15191) antibody 1:100 dilution), rabbit monoclonal anti-Anti-Cyclin D1 antibody [SP4] (ab16663) dilution 1:50) and rabbit monoclonal antibody Anti-p21 antibody [EPR3993] (ab109199), dilution 1:50), at 4uC ((Abcam, Cambridge, UK)). Hematoxylin stain was used to counter stain the nuclei. Positive controls were sections from lung carcinoma, breast carcinomas, and normal colon mucosa for COX2, cyclin D1 \& P21 respectively. Negative controls were done by omission of the primary antibodies replacing them by non-immune serum during the primary antibody incubation.

\section{2) Evaluation of COX2, cyclin D1 \& P21 immunostaining}

We have considered cytoplasmic expression as positive for COX2, and nuclear stain as positive for cyclin D1 \& P21 and were analyzed the results of staining semiquantitatively by the assessment of the staining intensity and extent of stain in tumor cells. We have scored extent of stained tumor cells as 0 if there are no stained tumor cells at all, 1 if $10 \%$ of tumor cells was stained, 2 if $11 \%-50 \%$ of the tumor cells were stained, 3 if $51 \%-80 \%$ of the tumor cells were stained, and 4 if $81 \%-100 \%$ of the tumor cells were stained. We have scored the intensity of the stain as 0 is if there were no stained tumor cells at all, as 1 if faint weak stain was noticed in tumor cells, 2 is moderate brown stain was noticed in tumor cells and 3 if strong brown stain was noticed in tumor cells. To reach the final immunohistochemistry stains score (IHS) we have multiplied values of both the intensity and the extent of the stain. A final IHS from $0-12$ is reached then the cut off value of 4 is used for adequate statistical analysis below which is low and 
above which is considered high expression of all markers [18].

\section{Statistical Analysis}

The categorical variables have been compared using Pearson's Chi-square test or Fisher's exact test. Overall Survival (OS) rate have been calculated as the time from diagnosis to death or the most recent follow-up contact. Relapse Free Survival (RFS) was calculated as the time from start of treatment to date of relapse or the most recent follow-up contact that patient was known as relapse free. Stratification of OS and RFS was done according markers. These time-to-event distributions were estimated using the method of Kaplan-Meier plot. All tests were two sided. A p-value $<0.05$ was considered significant. All statistics were performed using SPSS 22.0 for windows (SPSS Inc., Chicago, IL, USA).

\section{Results}

We have included $60 \mathrm{CRC}$ cases in our, their age range from 29 to 80 years (mean: $58.33 \pm 12.27$ years).

$36(60 \%)$ of patients were male and $24(40 \%)$ of cases were females. We included $52(86.7 \%)$ cases of conventional adenocarcinoma and $8(13.3 \%)$ cases of mucoid carcinoma, $13(21.7 \%)$ cases grade 1,31 (51.7\%) cases grade 2 and 16 (26.7\%) cases grade $3 \mathrm{DM}$ were detected in 17 cases (28.3\%).

The detailed demographic data of our patients are shown in Table 1.

\subsection{Cox-2 Immunoexpression and Its Correlation with Clinicopathological Features}

Cox-2 high expression was found in 31 (51.7\%) of cases and it was correlated with primary site of the tumor its expression was more when the tumor in the ascending colon $(0.034 \%)$, mucoid adenocarcinoma, larger tumor size, higher tumor grade, presence of lymph node\& distant metastasis, and advanced stage $(P=0.000)$. no detected significant difference regarding Cox-2 expression and sex of the patient (Table 2 and Figure 1).

\subsection{Cyclin D1 Immunoexpression and Its Correlation with Clinicopathological Features}

Cyclin D1 high expression was found in 29 (48.3\%) of cases and it was correlated with primary site of the tumor its expression was more when the tumor in the ascending colon $(\mathrm{P}<0.001)$, large tumor size, higher grade, presence of lymph node metastases $(P=0.000)$, distant metastasis $(p=0.029)$. no detected significant difference regarding Cyclin D1 expression, sex of the patient, or primary histopathology (Table 2 and Figure 2).

\subsection{P21 Immunoexpression and Its Correlation with Clinicopathological Features}

P21 high expression was found in 31 (51.7\%) and it was associated with conven- 
tional adenocarcinoma $(\mathrm{p}=0.017)$, smaller tumor size, lower grade, absence of lymph node metastases $(\mathrm{p}=0.002)$, early stage and absence of distant metastasis $(\mathrm{P}=0.001)$. no significant difference regarding $\mathrm{P} 21$ expression with sex of the patient (Table 2 and Figure 3).

Table 1. Clinicopathological features, immunohistochemical markers and outcome of our patients.

\begin{tabular}{|c|c|c|c|c|c|}
\hline \multirow{2}{*}{ Characteristics } & \multicolumn{2}{|c|}{ All patients $(\mathrm{N}=60)$} & \multirow{2}{*}{ Characteristics } & \multicolumn{2}{|c|}{ All patients $(\mathrm{N}=60)$} \\
\hline & No. & $\%$ & & No. & $\%$ \\
\hline$\underline{\text { Sex }}$ & & & $\underline{\text { Distant metastasis }}$ & & \\
\hline Male & 36 & $60 \%$ & Absent & 43 & $71.7 \%$ \\
\hline Female & 24 & $40 \%$ & Present & 17 & $28.3 \%$ \\
\hline Age year & & & $\underline{\text { Duke's stage }}$ & & \\
\hline Mean \pm SD & 58.33 & \pm 12.27 & Stage A & 14 & $23.3 \%$ \\
\hline Median (Range) & 60 & $(29-80)$ & Stage B & 11 & $18.3 \%$ \\
\hline$\underline{\text { Initial site }}$ & & & Stage C & 18 & $30 \%$ \\
\hline Ascending & 19 & $31.7 \%$ & Stage D & 17 & $28.3 \%$ \\
\hline Transverse & 6 & $10 \%$ & $\underline{\text { AJCC stage }}$ & & \\
\hline Descending & 5 & $8.3 \%$ & Stage I & 14 & $23.3 \%$ \\
\hline Rectosigmoid & 30 & $50 \%$ & Stage II & 12 & $20 \%$ \\
\hline$\underline{\text { Size }}$ & & & Stage III & 17 & $28.3 \%$ \\
\hline$\leq 5 \mathrm{~cm}$ & 27 & $45 \%$ & Stage IV & 17 & $28.3 \%$ \\
\hline$>5 \mathrm{~cm}$ & 33 & $55 \%$ & $\underline{\text { Response to treatment }}$ & & \\
\hline Pathological type & & & $\mathrm{PD}$ & 8 & $36.4 \%$ \\
\hline Conventional & 52 & $86.7 \%$ & SD & 6 & $27.3 \%$ \\
\hline Mucoid & 8 & $13.3 \%$ & PR & 3 & $13.6 \%$ \\
\hline Grade & & & CR & 5 & $22.7 \%$ \\
\hline Grade I & 13 & $21.7 \%$ & Follow-up months & & \\
\hline Grade II & 31 & $51.7 \%$ & Mean \pm SD & 23.11 & \pm 9.98 \\
\hline Grade III & 16 & $26.7 \%$ & Median (Range) & 25 & $(8-35)$ \\
\hline$\underline{\mathrm{T}}$ & & & $\underline{\text { Relepase }}$ & ( & \\
\hline $\mathrm{T} 1$ & 9 & $15 \%$ & Absent & 28 & $66.7 \%$ \\
\hline $\mathrm{T} 2$ & 14 & $23.3 \%$ & Present & 14 & $33.3 \%$ \\
\hline $\mathrm{T} 3$ & 12 & $20 \%$ & & & \\
\hline $\mathrm{T} 4$ & 25 & $41.7 \%$ & & & \\
\hline$\underline{\mathrm{LN} \text { metastasis }}$ & & & Death & & \\
\hline Node negative & 26 & $43.3 \%$ & Alive & 40 & $66.7 \%$ \\
\hline Node positive & 34 & $56.7 \%$ & Died & 20 & $33.3 \%$ \\
\hline
\end{tabular}

Response to treatment. PD; progressive disease, SD stable disease, PR partial response, CR complete response. 
A. M. Salem et al.

Table 2. Relation between COX2, Cyclin D1, p21 immunohistochemistry and clinicopathological parameters of our patients.

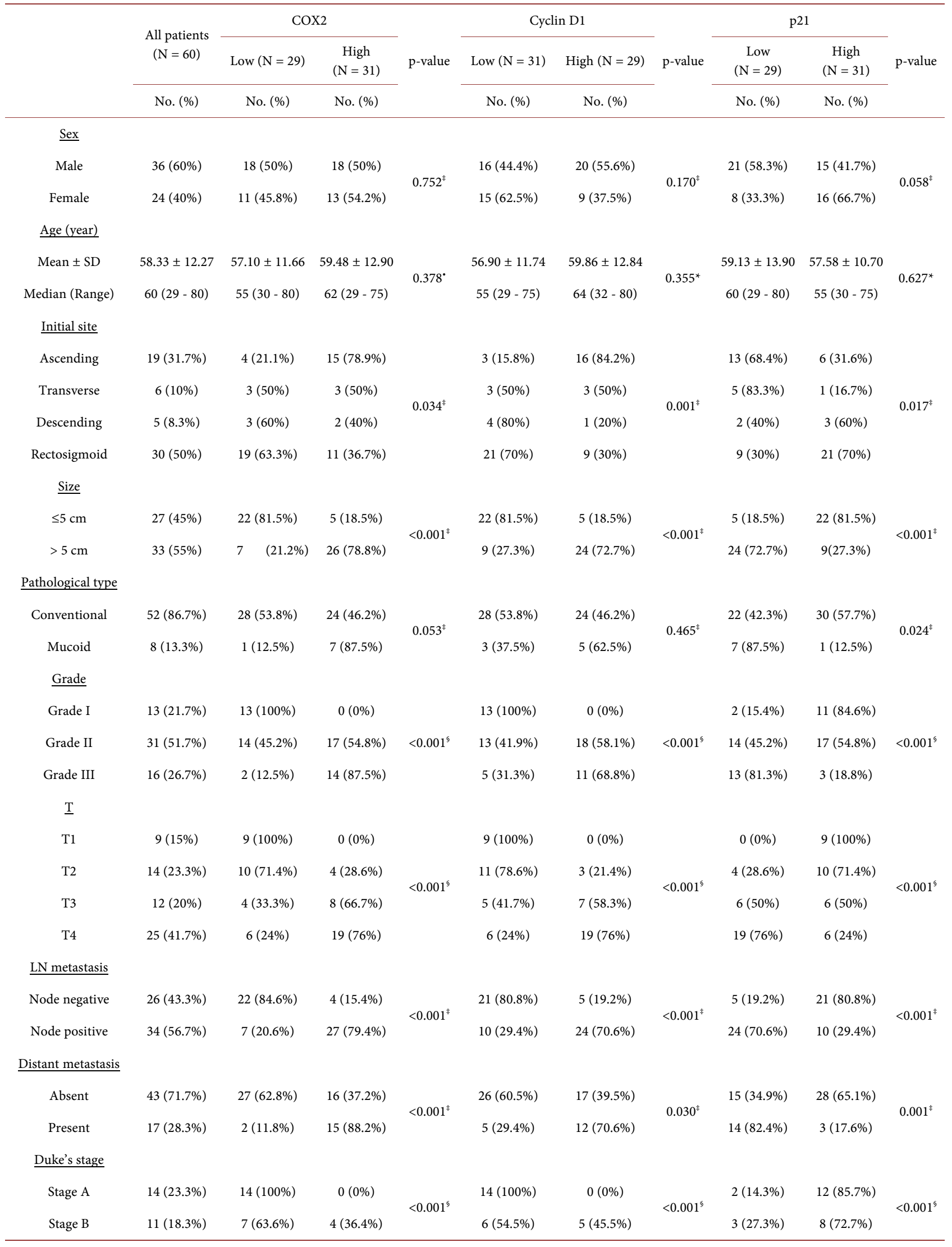




\section{Continued}

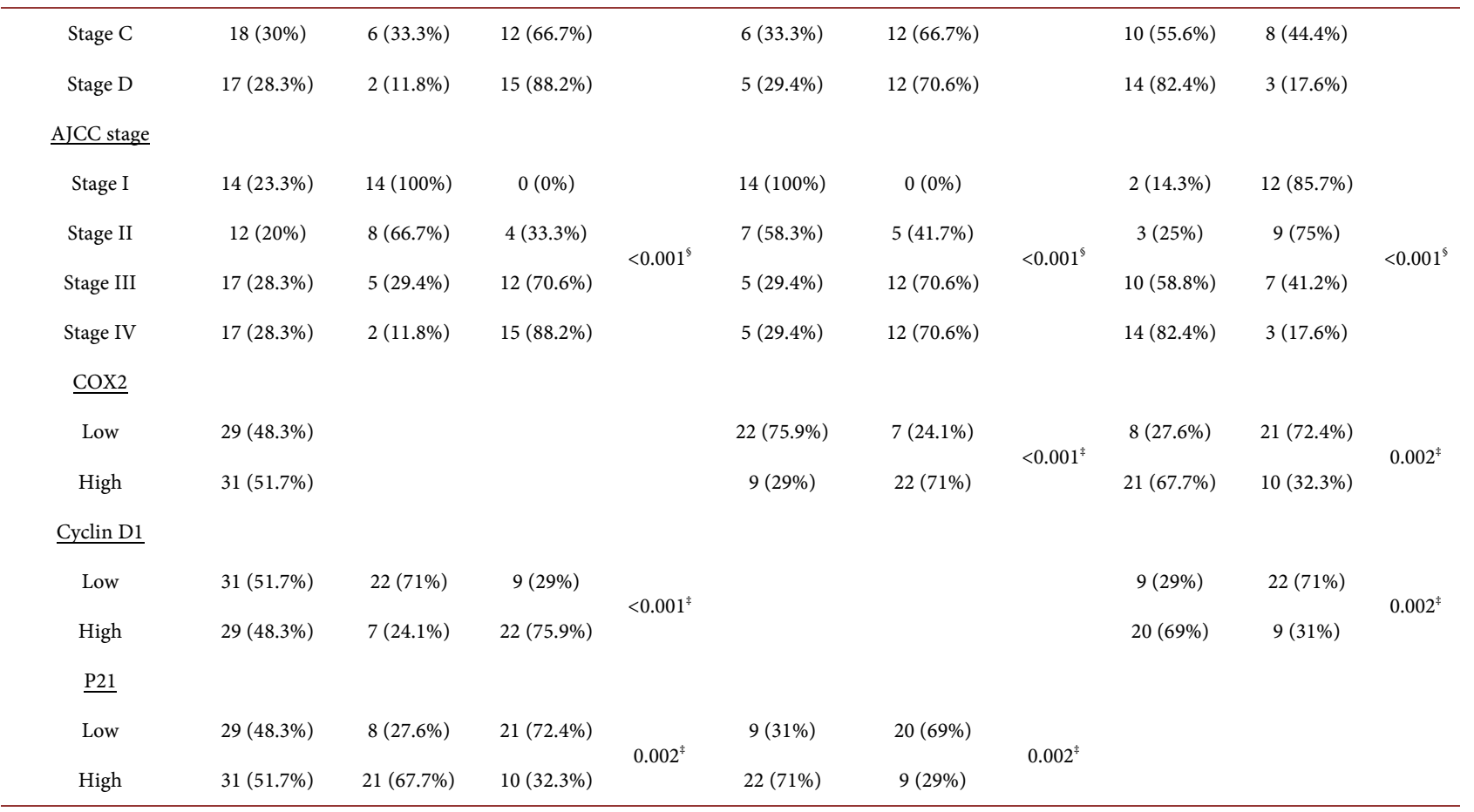

${ }^{*}$ Independent samples Student's t-test; ${ }^{\circ}$ Mann Whitney U test; ${ }^{\ddagger} \mathrm{Chi}$-square test; ${ }^{5} \mathrm{Chi}$-square test for trend; $\mathrm{p}<0.05$ is significant. A final IHS from 0 - 12 is reached then the cut off value of 4 is used for adequate statistical analysis below which is low and above which is considered high expression of all markers.

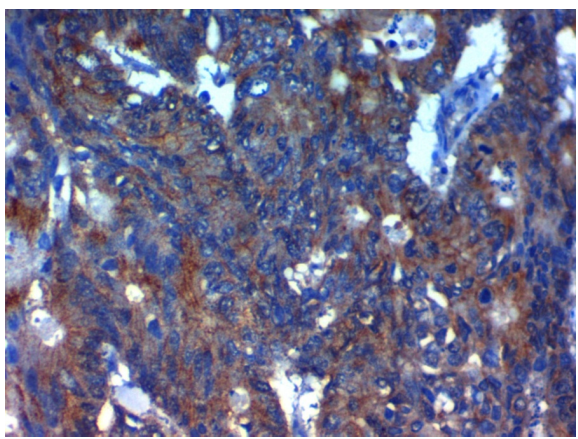

(a)

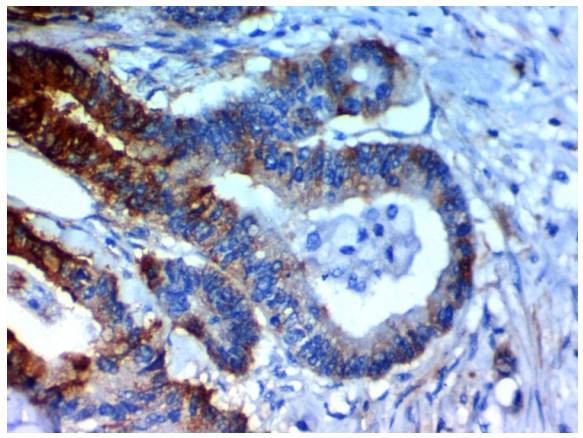

(c)

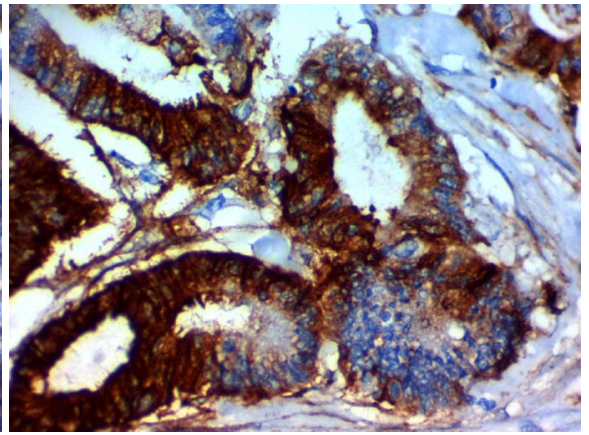

(b)

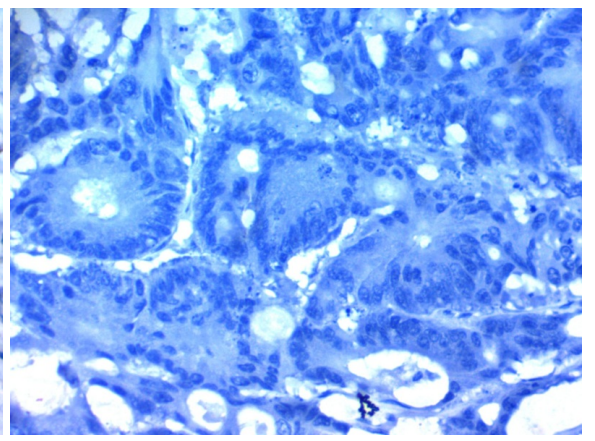

(d)

Figure 1. Immunohistochemical expression of Cox-2 in colorectal carcinoma (CRC). (a) High expression in the cytoplasm of poorly differentiated CRC $\times 400$, (b) High expression in the cytoplasm of moderately differentiated CRC $\times 400$, (c) low expression in the cytoplasm of well differentiated CRC $\times 400$, (d) negative expression in the cytoplasm of well differentiated CRC $\times 400$. Cox- 2 stains \& Hematoxylin counter stain. 


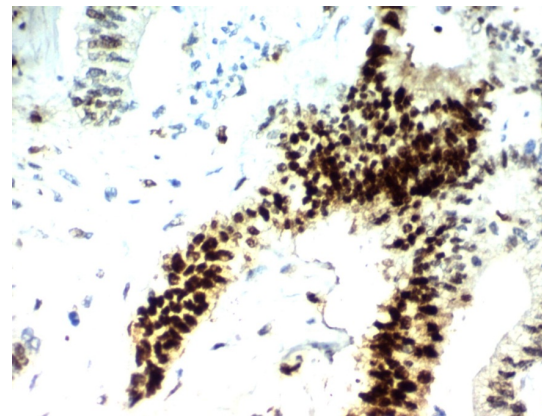

(a)

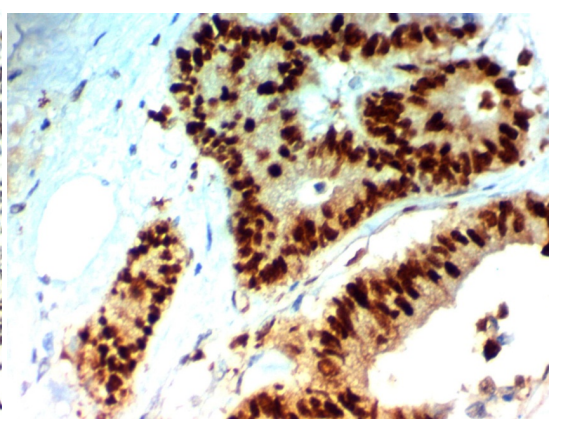

(b)

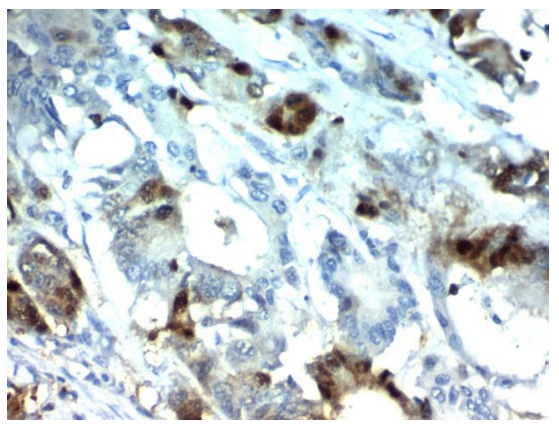

(c)

Figure 2. Immunohistochemical expression of Cyclin D1 in colorectal carcinoma (CRC). (a) High expression in the nucleus of poorly differentiated CRC $\times 400$, (b) High expression in the nucleus of moderately differentiated CRCx400, (c) Low expression in the nucleus of well differentiated CRC $\times 400$. Cyclin D-1stain\& Hematoxylin counter stain.

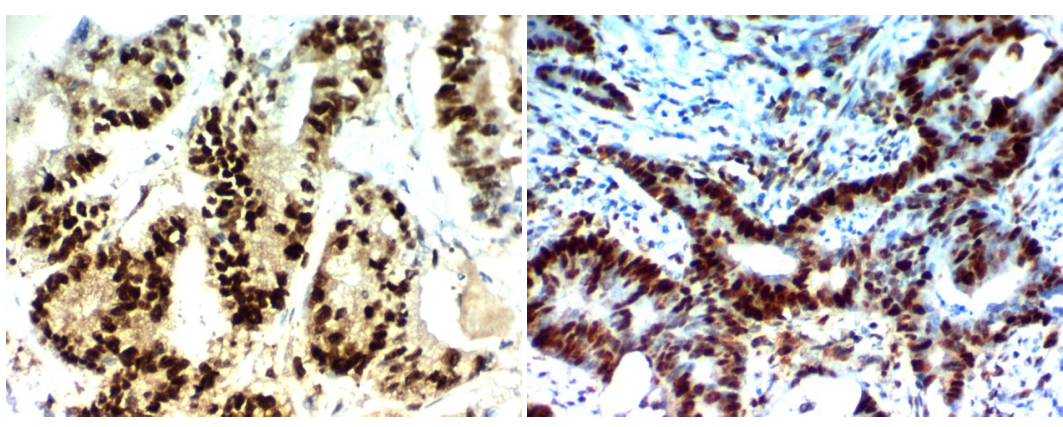

(a) (b)

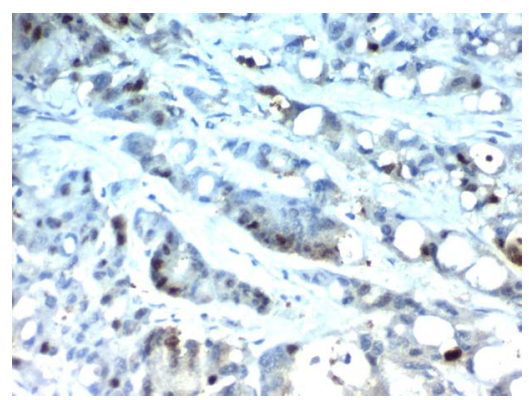

(c)

Figure 3. Immunohistochemical expression of P21 in colorectal carcinoma (CRC). (a) High expression in the nucleus of well differentiated CRC $\times 400$, (b) High expression in the nucleus of moderately differentiated CRC $\times 400$, (c) Low expression in the nucleus of poorly differentiated CRC $\times 400$. P21 stains Hematoxylin counter stain. 


\subsection{Correlation between Immunohistochemical Expression of Cox-2, Cyclin D1 and P21 in CRC}

We found a direct relationship between Cox-2 and Cyclin D1, an inverse relationship between Cox-2 and P21, and an inverse relationship between Cyclin D1 and $\mathrm{P} 21(\mathrm{P}<0.001)$.

\subsection{Follow-Up Results}

Combination of overexpression of Cox-2 \& Cyclin D1 and decresed expression of P21 is positively correlated with higher incidence of CRC recurrence after successive therapy $(\mathrm{P}=0.04,0.000$ respectively), shorter OS\& RFS rates $(\mathrm{p}=$ $0.002,0.004 \& 0.000$ respectively) but no significant correlation with response to therapy with all markers (Table 3 and Figure 4 \& Figure 5).

Table 3. Relation between COX2, Cyclin D1, p21 immunohistochemistry and outcome of treatment of our patients.

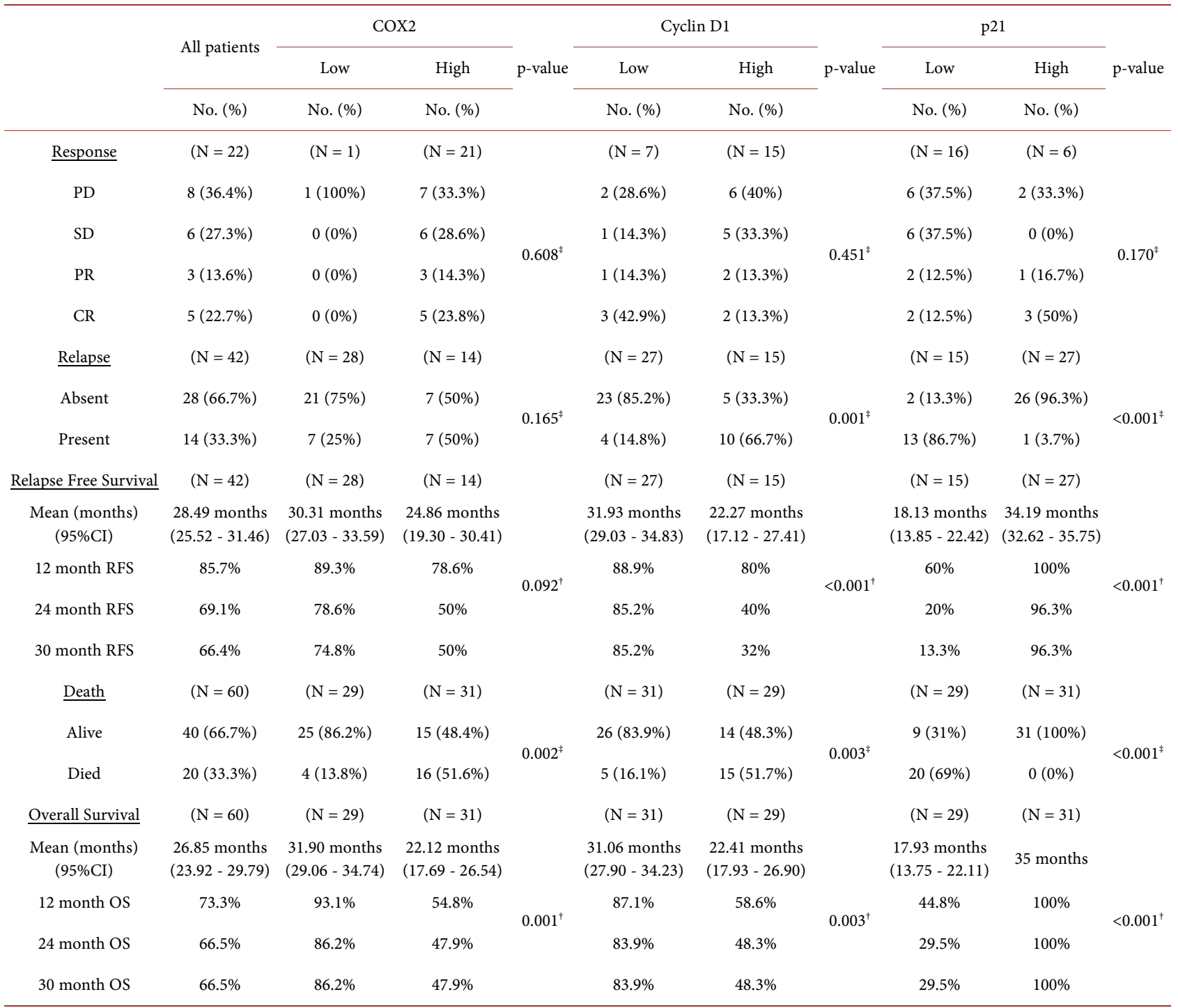

${ }^{\ddagger}$ Chi-square test; ${ }^{\dagger} \log$ rank test; $\mathrm{p}<0.05$ is significant. A final IHS from $0-12$ is reached then the cut off value of 4 is used for adequate statistical analysis below which is low and above which is considered high expression of all markers. 

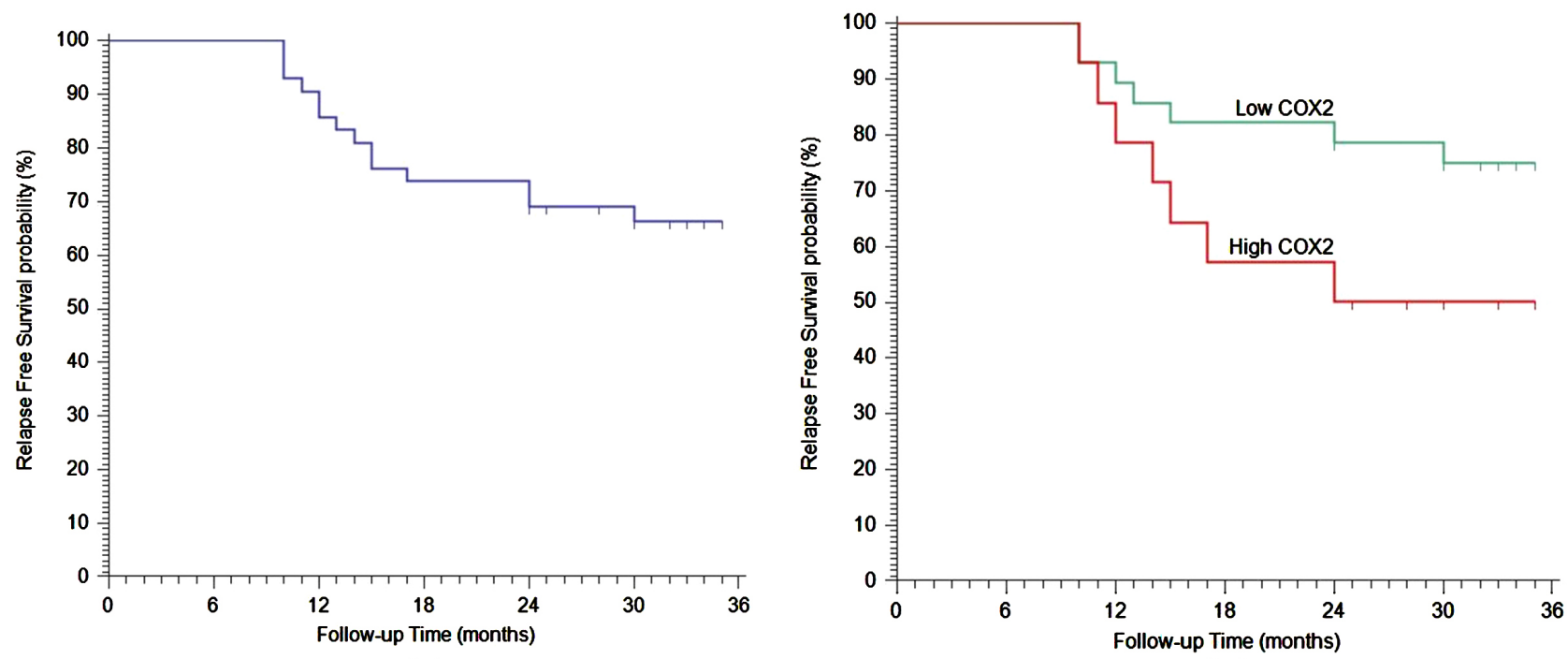

(a)

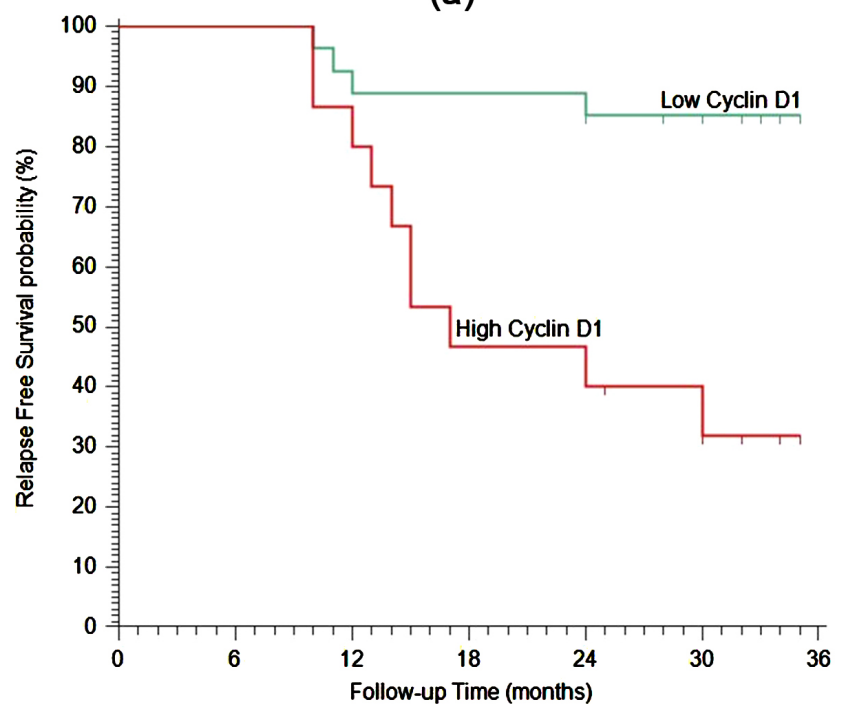

(c)

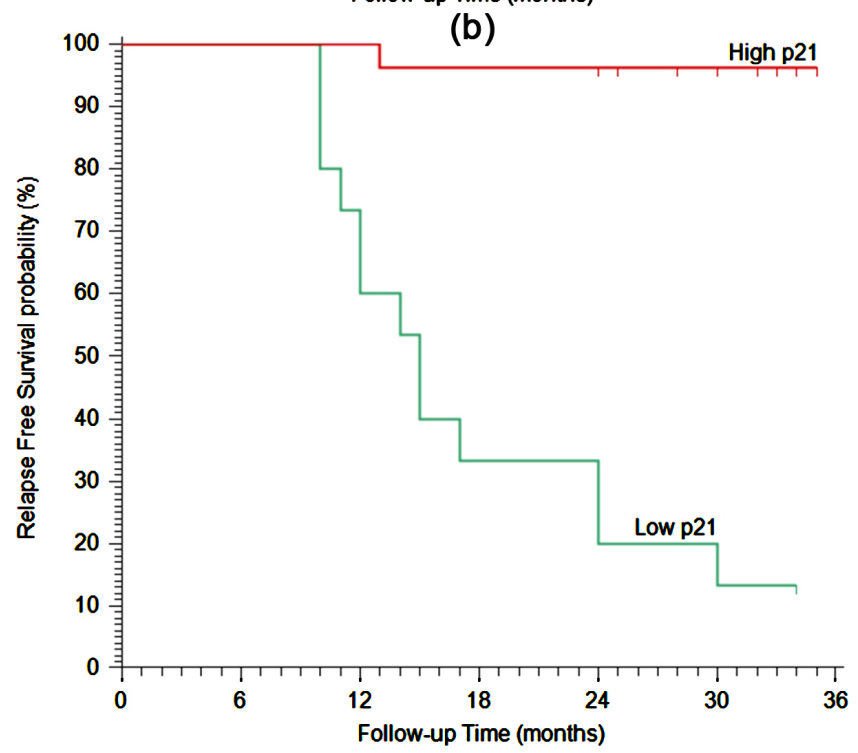

(d)

Figure 4. Kaplan Meir survival curves of Recurrence free survival (RFS) of the studied colorectal carcinoma (CRC) patients (a) RFS rate of all the studied CRC cases (b) RFS rate stratified according to Cox-2 expression (c) RFS rate stratified according to Cyclin D1 expression (d) RFS rate stratified according to P21 expression.

\section{Discussion}

CRC is considered a major health problem especially in developing countries and it seriously affects public health, as despite improvements in management and treatment modalities the 5 year OS rate of CRC patients is still disappointing [4]. Detection of new targeted therapies is the hope to improve the prognosis.

Increased COX-2 expression is found in cancer of many organs and related to dismal outcome e.g. cancer lung, cancer cervix and esophageal squamous cell carcinoma [19]. As its role in CRC is still not clarified we evaluated its expression.

In our study we proved that Cox-2 high expression was found in 31 (51.7\%) of cases and it was correlated with primary site of the tumor its expression was 


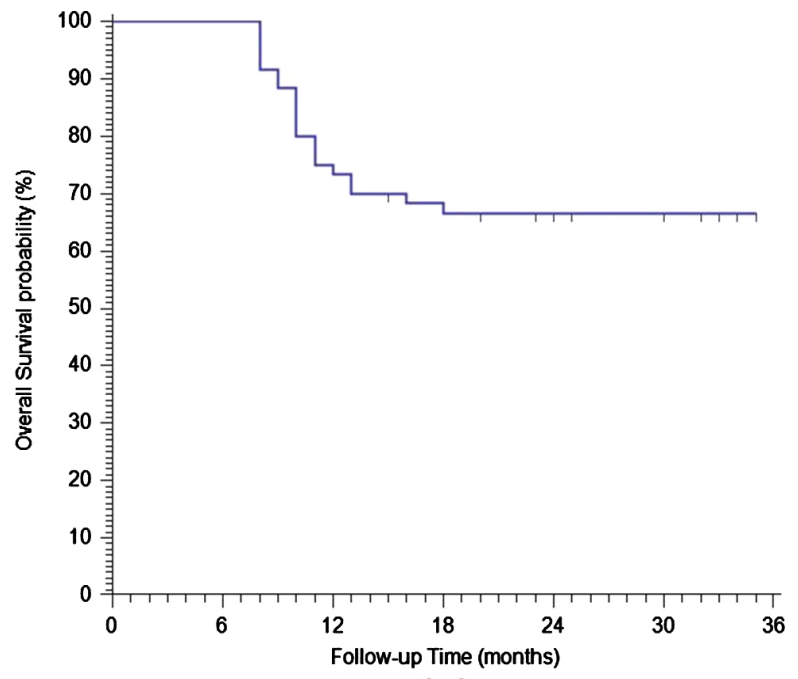

(a)

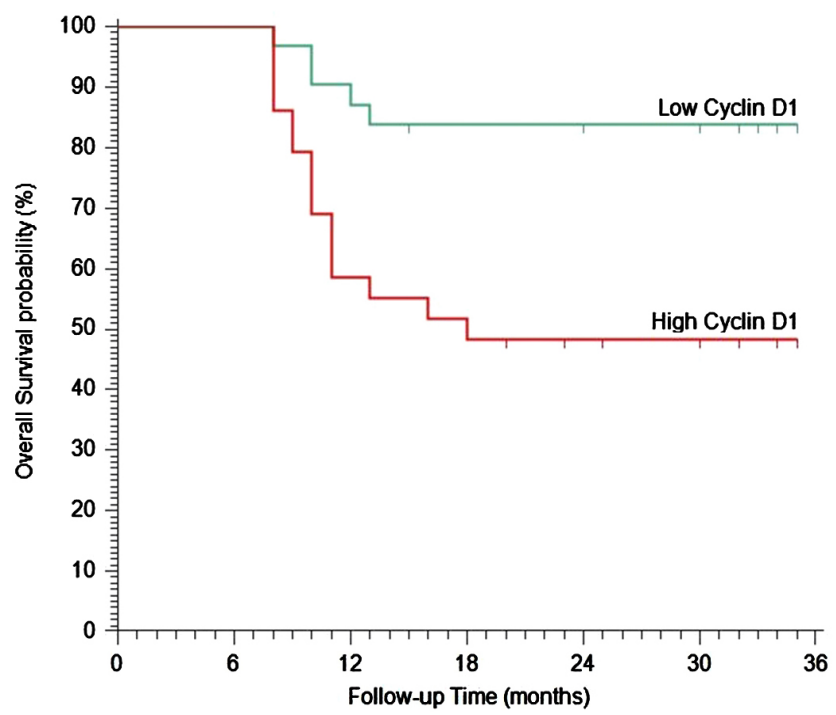

(c)

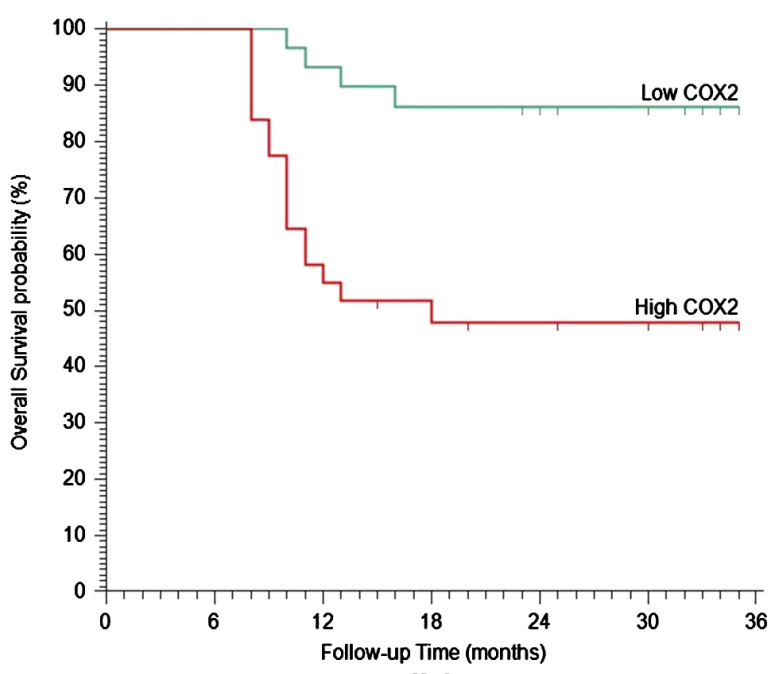

(b)

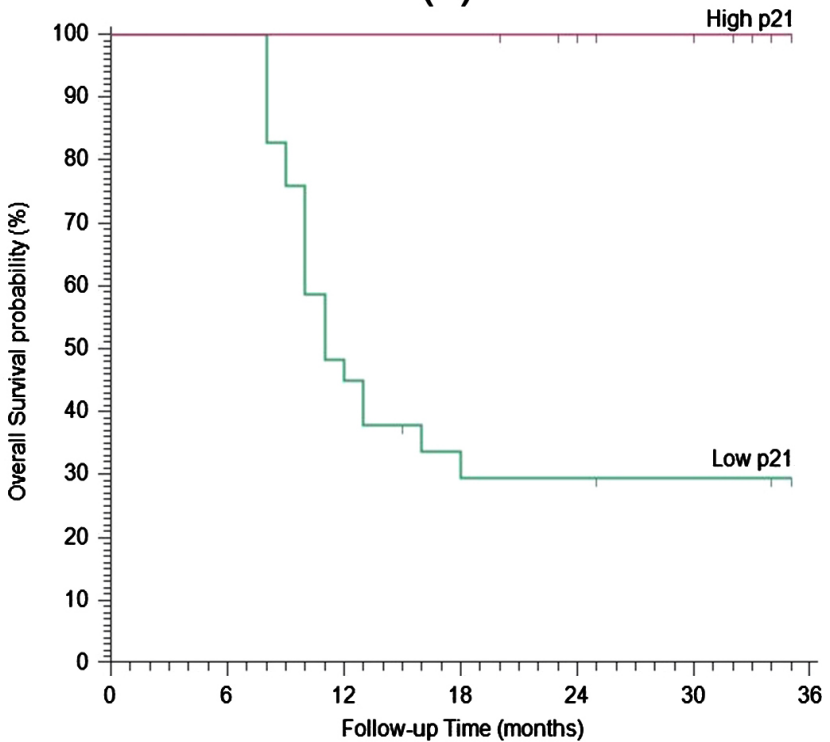

(d)

Figure 5. Kaplan Meir survival curves of overall survival rate (OS) of the of the studied colorectal carcinoma (CRC) patients (a) OS rate of all the studied CRC cases (b) OS rate stratified according to Cox-2 expression (c) OS rate stratified according to Cyclin D1 expression (d) OS rate stratified according to P21 expression.

more when the tumor in the ascending colon $(0.034 \%)$, mucoid adenocarcinoma, larger tumor size, higher tumor grade, presence of lymph node\& distant metastasis, and advanced stage $(\mathrm{P}=0.000)$, similarly, Elzagheid et al, [4]. reported increased expression of COX-2 in CRC and reported 74\% of CRCs expressed COX-2, also Wu et al., [20] have shown the same results that COX2 was expressed in $85 \%$ - 95\% of CRCs. Similar to our results, Al-Maghrabi et al. [21] found that $56 \%$ of primary CRC cases expressed COX2 and its expression was related to poor clinicopathological parameters and poor patients prognosis, which clarified its important role in CRC progression.

The discrepancies between the different studies may be due to variability in patients' number and their characteristics, use of different antibodies clones, 
different methods of evaluation of the stainin and differences in the cut-off levels of the evaluated slides. Similar to our results COX2 immunostaining was cytoplasmic in CRC and the colonic epithelium that is adjacent to the tumor revealed negative stain for COX2 [22].

The positive correlation between COX2 expression, higher tumor grade and advanced stage revealed that COX-2 could be used as targeted therapy and prognostic marker for patients with CRC that support the view that COX2 plays an important role in advanced CRC. These finding in agreement with; Elzagheid et al. [4]. who found higher expression of COX2 in advanced-stage than in early-stage CRC. Also Lim et al. [23], proved that increased COX2 expression was related to depth of invasion and advanced stage.

We have found that there is higher COX2 expression in CRC located in the ascending colon (0.034). Different results were found by Peng et al., 2013 that there were higher levels of COX-2 expression in patients with rectal cancer compared to patients whose tumors were located in the colon, they explained that by local variability in regulatory factors that are responsible for COX-2 expression. The role of Cox-2 in CRC carcinogenesis may be due to its role in inflammation and subsequent disturbances in cell cycle progression in malignant cells [4]. so we have evaluated its expression with 2 markers that are involved in cell cycle control; CyclinD1 and P21.

In this study, we found that Cyclin D1 high expression was found in 29 (48.3\%) of cases and it was correlated with primary site of the tumor its expression was more when the tumor in the ascending colon $(\mathrm{P}<0.001)$, large tumor size, higher grade, presence of lymph node metastases $(P=0.000)$, distant metastasis $(\mathrm{p}=0.029)$. similarly, Van Wangenheim et al. [14], who found that cyclin D1 was correlated poor clinicopathological criteria of cancer. Also, Holland et al. [24] have proved increased Cyclin D1nuclear expression in the right colon that was similar to our results. However, such association has not been found with cytoplasmic expression. Controversially Wong et al. [25] and McKay et al., [26] evaluated the association between marker expression and clinicopathological criteria without associations with its subcellular localization.

Our findings in this study agreed with Wangefjord et al. [27], who noted important prognostic role of in patients with stage III-IV CRC but they did not find any association in patients with early stage I-II CRC, also Bahnassy et al. [28] and Maeda et al. [29] have reported that increased cyclin D1 expression has been related to dismal prognosis. In contrast to our results, Holland et al. [24] \& Ogino et al. [15] have concluded that cyclin Dloverexpression is related to good prognosis additionally they have found that cyclin D1-expressing tumors are less aggressive than tumors with decreased cyclin D1 expression. Moreover Lehn et al. [30] stated the same results in breast cancer, and proved an association between decreased cyclin D1 expression levels and invasive cancer phenotype. The favourable prognostic role of Cyclin D1 in previous studies could be explained by that higher cyclin D1 expressing tumors showed better response to adjuvant chemotherapy this is the cause of difference with our study as we have not find a 
significant relations between Cyclin D1 expression and reponse to chemotherapy. Van Wangenheim et al. [14] and Holland et al. [24] reported that cyclin D1 overexpression was correlated with longer patients' survival; these differences may be due to differences in methods and procedures of staining and evaluation.

In our study there is no statistically significant difference between Cyclin D1and genders this different from results of Wangefjord et al., [27] who revealed that the prognostic value of cyclin D1 was only evident in male but not female CRC patients, a finding that has, not been reported previously. It will be of beneficial studies to detect the molecular pathogenesis for this prognostic significance of cyclin D1 in women and men that might be referred to the effect of sex-hormone levels, genetic and epigenetic modification of steroid receptors.

P21 high expression was found in $31(51.7 \%)$ and it was associated with conventional adenocarcinoma $(p=0.017)$, smaller tumor size,, lower grade, absence of lymph node metastases $(p=0.002)$, early stage and absence of distant metastasis $(\mathrm{P}=0.001)$. These finding were in agreement with Ogino et al. [15] who found that $\mathrm{p} 21$ loss in tumor cells was associated with longer survival because of the function of p21 appears to be tumor-suppressive [15]. P21 which is a cyclin-dependent kinase inhibitor that is affected by p 53 wild-type, plays an essential role in blocking of the cell cycle progression [16].

But p21 could facilitate malignant cells invasion by p21-activated kinase-1 (PAK1) in melanoma cells [31]. Previous studies stated p21 loss can be a marker for aggressive tumor that was similar to our results [32].

Additionally, Esposito et al., [33], stated that lung cancer patients that were negative for $\mathrm{p} 21$ had shorter OS rate than those with high P21 expression that was similar to our results in CRC.

\section{Summery \& Conclusions}

We proved that combination of Cox-2 \& Cyclin D1 overexpression in addition to P21 low expression is considered markers of poor prognosis in CRC patients, mainly due to disturbances in cell cycle control. Future studies that include combination of gene study of these markers in addition to their tissue protein expression are recommended to prove our results.

\section{Conflicts of Interest}

The authors declare no conflicts of interest regarding the publication of this paper.

\section{References}

[1] Favoriti, P., Carbone, G., Greco, M., et al. (2016) Worldwide Burden of Colorectal Cancer: A Review. Updates in Surgery, 68, 7-11. https://doi.org/10.1007/s13304-016-0359-y

[2] Rafiemanesh, H., Pakzad, R., Abedi, M., et al. (2016) Colorectal Cancer in Iran: Epidemiology and Morphology Trends. EXCLI Journal, 15, 738-744. 
[3] Wood, L.D., Parsons, D.W., Jones, S., et al. (2007) The Genomic Landscapes of Human Breast and Colorectal Cancers. Science, 318, 1108-1113. https://doi.org/10.1126/science.1145720

[4] Elzagheid, A., Emaetigi, F., Lamia Alkikhia, L., et al. (2013) High Cyclooxygenase-2 Expression Is Associated with Advanced Stages in Colorectal Cancer. Anticancer Research, 33, 3137-3144.

[5] Li, Y., Wei, J., Xu, C., et al. (2014) Prognostic Significance of Cyclin D1 Expression in Colorectal Cancer: A Meta-Analysis of Observational Studies. PLOS ONE, 9, e94508. https://doi.org/10.1371/journal.pone.0094508

[6] Cao, J., Einstein, M., Anderson, P., et al. (2002) Expression of COX-2, Ki-67, Cyclin D1, and P21 in Endometrial Endometrioid Carcinomas. International Journal of Gynecological Pathology, 21, 147-154. https://doi.org/10.1097/00004347-200204000-00007

[7] Peng, L., Zhou, Y., Wang, Y., et al. (2013) Prognostic Significance of COX-2 Immunohistochemical Expression in Colorectal Cancer: A Meta-Analysis of the Literature. PLOS ONE, 8, e58891.

[8] Wu, W.K., Sung, J.J., Lee, C.W., et al. (2010) Cyclooxygenase-2 in Tumorigenesis of Gastrointestinal Cancers: An Update on the Molecular Mechanisms. Cancer Letters, 295, 7-16. https://doi.org/10.1016/j.canlet.2010.03.015

[9] Tatsuguchi, A., Kishida, T., Fujimori, S., et al. (2006) Differential Expression of Cyclo-Oxygenase-2 and Nuclear B-Catenin in Colorectal Cancer Tissue. Alimentary Pharmacology \& Therapeutics, 24, 153-159.

https://doi.org/10.1111/j.1365-2036.2006.00039.x

[10] Jiang, H., Wang, J. and Zhao, W. (2013) Cox-2 in Non-Small Cell Lung Cancer: A Meta-Analysis. Clinica Chimica Acta, 419, 26-32. https://doi.org/10.1016/j.cca.2013.01.012

[11] Song, J., Su, H., Zhou, Y., et al. (2014) Cyclooxygenase-2 Expression Is Associated with Poor Overall Survival of Patients with Gastric Cancer: A Meta-Analysis. Digestive Diseases and Sciences, 59, 436-445. https://doi.org/10.1007/s10620-013-2917-1

[12] Theocharis, S., Giaginis, C., Parasi, A., et al. (2007) Expression of Peroxisome Proliferator-Activated Receptor- $\gamma$ in Colon Cancer: Correlation with Histopathological Parameters, Cell Cycle-Related Molecules, and Patients' Survival. Digestive Diseases and Sciences, 52, 2305-2311. https://doi.org/10.1007/s10620-007-9794-4

[13] Besson, A., Dowdy, S.F. and Roberts, J.M. (2008) CDK Inhibitors: Cell Cycle Regulators and Beyond. Developmental Cell, 14, 159-169. https://doi.org/10.1016/j.devcel.2008.01.013

[14] Van Wangenheim, C., Monig, S., Schneider, P., et al. (2008) p16, Cyclin D1 and Rb Expression in Colorectal Carcinomas: Correlations with Clinico-Pathological Parameters and Prognosis. Molecular Medicine Reports, 1, 27-32.

[15] Ogino, S., Nosho, K., Irahara, N., et al. (2009) A Cohort Study of Cyclin D1 Expression and Prognosis in 602 Colon Cancer Cases. Clinical Cancer Research, 15, 4431-4438. https://doi.org/10.1158/1078-0432.CCR-08-3330

[16] Abukhdeir, A.M. and Park, B.H. (2008) P21 and p27 Roles in Carcinogenesis and Drug Resistance. Expert Reviews in Molecular Medicine, 10, e19. https://doi.org/10.1017/S1462399408000744

[17] Hsu, S.M., Raine, L. and Fanger, H. (1981) Use of Avidin-Biotin-Peroxidase Complex $(\mathrm{ABC})$ in Immunoperoxidase Techniques: A Comparison between $\mathrm{ABC}$ and Unlabeled Antibody (PAP) Procedures. Journal of Histochemistry \& Cytochemi- 
stry, 29, 577-580. https://doi.org/10.1177/29.4.6166661

[18] Bagir, E.K., Açikalin, A., Izol, V., Seydaoglu, G. and Erdogan, S. (2016) Immunohistochemical Study of Cyclooxygenase-2 Expression in Prostate Carcinoma: It's Relation with Apoptosis and Angiogenesis. Bulletin of Urooncology, 15, 144-147.

[19] Lee, J.Y., Myung, S.K. and Song, Y.S. (2013) Prognostic Role of Cyclooxygenase-2 in Epithelial Ovarian Cancer: A Meta-Analysis of Observational Studies. Gynecologic Oncology, 129, 613-619. https://doi.org/10.1016/j.ygyno.2013.02.011

[20] Wu, A.W., Gu, J., Ji, J.F., Li, Z.F. and Xu, G.W. (1990) Role of COX2 in Carcinogenesis of Colorectal Cancer and Its Relationship with Tumor Biological Characteristics and Patients' Prognosis. World Journal of Gastroenterology, 9, 1990-1994.

[21] Al-Maghrabi, J., Buhmeida, A., Emam, E., et al. (2012) Cyclooxygenase-2 Expression as a Predictor of Outcome in Colorectal Carcinoma. World Journal of Gastroenterology, 18, 1793-1799. https://doi.org/10.3748/wjg.v18.i15.1793

[22] Miladi-Abdennadher, I., Abdelmaksoud-Dammak, R., Ayed-Guerfali, D.B., et al. (2012) Expression of COX2 and E-Cadherin in Tunisian Patients with Colorectal Adenocarcinoma. Acta Histochemica, 114, 577-581. https://doi.org/10.1016/j.acthis.2011.11.002

[23] Lim, S.C., Cho, H., Lee, T.B., et al. (2010) Impacts of Cytosolic Phospholipase A2, 15-Prostaglandin Dehydrogenase, and Cyclooxygenase-2 Expressions on Tumor Progression in Colorectal Cancer. Yonsei Medical Journal, 51, 692-699. https://doi.org/10.3349/ymj.2010.51.5.692

[24] Holland, T.A., Elder, J., McCloud, J.M., et al. (2001) Subcellular Localisation of Cyclin D1 Protein in Colorectal Tumours Is Associated with p21 (WAF1/CIP1) Expression and Correlates with Patient Survival. International Journal of Cancer, 95, 302-306. https://doi.org/10.1002/1097-0215(20010920)95:5<302::AID-IJC1052>3.0.CO;2-\#

[25] Wong, N.A., Morris, R.G., McCondochie, A., et al. (2002) Cyclin D1 Overexpression in Colorectal Carcinoma in Vivo Is Dependent on Beta-Catenin Protein Dysregulation, But Not k-Ras Mutation. The Journal of Pathology, 197, 128-135. https://doi.org/10.1002/path.1113

[26] McKay, J.A., Douglas, J.J., Ross, V.G., et al. (2002) Analysis of Key Cell-Cycle Checkpoint Proteins in Colorectal Tumours. The Journal of Pathology, 196, 386-393. https://doi.org/10.1002/path.1053

[27] Wangefjord, S., Manjer, J., Gaber, A., et al. (2010) Cyclin D1 Expression in Colorectal Cancer Is a Favorable Prognostic Factor in Men But Not in Women in a Prospective, Population-Based Cohort Study. Biology of Sex Differences, 2, 10. http://www.bsd-journal.com/content/2/1/10

[28] Bahnassy, A.A., Zekri, A.R., El-Houssini, S., et al. (2004) Cyclin A and Cyclin D1 as Significant Prognostic Markers in Colorectal Cancer Patients. BMC Gastroenterology, 4, 22. https://doi.org/10.1186/1471-230X-4-22

[29] Maeda, K., Chung, Y.S., Kang, S.M., et al. (1997) Overexpression of Cyclin D1 and p53 Associated with Disease Recurrence in Colorectal Adenocarcinoma. International Journal of Cancer, 74, 310-315.

https://doi.org/10.1002/(SICI)1097-0215(19970620)74:3<310::AID-IJC13>3.0.CO;2F

[30] Lehn, S., Tobin, N.P., Berglund, P., et al. (2010) Down-Regulation of the Oncogene Cyclin D1 Increases Migratory Capacity in Breast Cancer and Is Linked to Unfavorable Prognostic Features. American Journal of Pathology, 177, 2886-2897. https://doi.org/10.2353/ajpath.2010.100303 
[31] Pavey, S., Zuidervaart, W., van Nieuwpoort, F., et al. (2006) Increased p21-Activated Kinase-1 Expression Is Associated with Invasive Potential in Uveal Melanoma. $\mathrm{Me}$ lanoma Research, 16, 285-296. https://doi.org/10.1097/01.cmr.0000222589.30117.f2

[32] He, H. and Baldwin, G.S. (2008) GTPases and p21-Activated Kinase in the Regulation of Proliferation and Apoptosis by Gastrins. The International Journal of Biochemistry \& Cell Biology, 40, 2018-2022.

https://doi.org/10.1016/j.biocel.2008.05.002

[33] Esposito, V., Baldi, A., Tonini, G., et al. (2004) Analysis of Cell Cycle Regulator Proteins in Non-Small Cell Lung Cancer. Journal of Clinical Pathology, 57, 58-63. https://doi.org/10.1136/jcp.57.1.58 